\title{
A Large-Scale Chemical Screen for Regulators of the Arginase 1 Promoter Identifies the Soy Isoflavone Daidzein as a Clinically Approved Small Molecule That Can Promote Neuronal Protection or Regeneration via a cAMP-Independent Pathway
}

\author{
Thong C. Ma, ${ }^{1 *}$ Aline Campana, ${ }^{2 *}$ Philipp S. Lange, ${ }^{1,8}$ Hsin-Hwa Lee, ${ }^{1}$ Kasturi Banerjee, ${ }^{1}$ J. Barney Bryson, ${ }^{2}$ \\ Lata Mahishi, ${ }^{1}$ Shabnam Alam, ${ }^{6}$ Roman J. Giger, ${ }^{3,6}$ Stephen Barnes, ${ }^{7}$ Sidney M. Morris Jr., ${ }^{4}$ Dianna E. Willis, ${ }^{5}$ \\ Jeffrey L. Twiss, ${ }^{5}$ Marie T. Filbin, ${ }^{2}$ and Rajiv R. Ratan ${ }^{1}$ \\ ${ }^{1}$ Burke-Cornell Medical Research Institute, White Plains, New York 10605, ${ }^{2}$ Biology Department, Hunter College, New York, New York 10021, ${ }^{3}$ University \\ of Michigan School of Medicine, Ann Arbor, Michigan 48109, ${ }^{4}$ University of Pittsburgh School of Medicine, Pittsburgh, Pennsylvania 15261, 5 Nemours \\ Biomedical Research, Alfred I. duPont Hospital for Children, Wilmington, Delaware 19899, ${ }^{6}$ University of Rochester, Rochester, New York 14642 , \\ ${ }^{7}$ University of Alabama Birmingham, Birmingham, Alabama 35294, and ${ }^{8}$ Department of Anesthesiology and Intensive Care Medicine, University of Bonn, \\ 53127 Bonn, Germany
}

An ideal therapeutic for stroke or spinal cord injury should promote survival and regeneration in the CNS. Arginase 1 (Arg1) has been shown to protect motor neurons from trophic factor deprivation and allow sensory neurons to overcome neurite outgrowth inhibition by myelin proteins. To identify small molecules that capture Argl's protective and regenerative properties, we screened a hippocampal cell line stably expressing the proximal promoter region of the arginase 1 gene fused to a reporter gene against a library of compounds containing clinically approved drugs. This screen identified daidzein as a transcriptional inducer of Arg1. Both CNS and PNS neurons primed in vitro with daidzein overcame neurite outgrowth inhibition from myelin-associated glycoprotein, which was mirrored by acutely dissociated and cultured sensory neurons primed in vivo by intrathecal or subcutaneous daidzein infusion. Further, daidzein was effective in promoting axonal regeneration in vivo in an optic nerve crush model when given intraocularly without lens damage, or most importantly, when given subcutaneously after injury. Mechanistically, daidzein requires transcription and induction of Argl activity for its ability to overcome myelin inhibition. In contrast to canonical Arg1 activators, daidzein increases Arg1 without increasing CREB phosphorylation, suggesting its effects are cAMP-independent. Accordingly, it may circumvent known CNS side effects of some cAMP modulators. Indeed, daidzein appears to be safe as it has been widely consumed in soy products, crosses the blood- brain barrier, and is effective without pretreatment, making it an ideal candidate for development as a therapeutic for spinal cord injury or stroke.

\section{Introduction}

Despite significant focus on neuroprotection, stroke and spinal cord injury (SCI) remain prevalent causes of disability worldwide as therapeutics designed to limit cell death have failed to enhance recovery in humans (Baptiste and Fehlings, 2007; Savitz and

\footnotetext{
Received 0ct. 23, 2009; revised Nov. 19, 2009; accepted Nov. 24, 2009.

This work was supported by National Institutes of Health (NIH) Grants NS040591 to R.R.R. and GM057384, a New York State Spinal Cord Injury Research Board Center of Research Excellence Grant to R.R.R., M.T.F., and R.J.G. (C019772), Adelson Foundation grants to R.R.R. and J.L.T., the Purdue University-University of Alabama at Birmingham Botanicals (enter for Age-Related Disease grant from the National Center for Complementary and Alternative Medicine and the NIH Office of Dietary Supplements (P50 AT00477, C. Weaver, Principal Investigator), and the Wings for Life Foundation. We thank Dr. Alvaro Estévez and Dr. Marco Domeniconi for their assistance with this manuscript. *T.C.M. and A.C. contributed equally to this manuscript.

Correspondence should be addressed to Dr. Rajiv R. Ratan, Burke-Cornell Medical Research Institute, 785 Mamaroneck Avenue, White Plains, NY 10605. E-mail: rratan@burke.org.

DOI:10.1523/JNEUROSCI.5266-09.2010

Copyright $\odot 2010$ the authors $\quad 0270-6474 / 10 / 300739-10 \$ 15.00 / 0$
}

Fisher, 2007; Ratan and Noble, 2009). As such, therapies that both protect neurons and foster CNS repair may facilitate progress beyond the limited benefits of currently available agents. Functional recovery after injury requires many barriers to be surmounted, including inhibitory myelin proteins (Cafferty and Strittmatter, 2006; Hannila and Filbin, 2008). Indeed, direct neutralization of these proteins has resulted in improved recovery after injury (Rossignol et al., 2007). Alternatively, neurons can be induced to "ignore" inhibitory cues activated by myelin (Cai et al., 1999).

Paradigms to develop novel neuroprotectants that also target CNS repair have come from prior elegant studies. "Priming" of DRG neurons by conditioning peripheral lesion or with neurotrophic factors allows neurons to resist myelin inhibitors; both mediate their beneficial effects by increasing cAMP (Filbin, 2003). Excitingly, agents that enhance cAMP levels promote axonal regeneration in injury models (Neumann et al., 2002; Qiu et 
al., 2002; Lu et al., 2004; Nikulina et al., 2004; Pearse et al., 2004). These regenerative effects are transcription-dependent and are mediated by arginase 1 (Arg1), a notable gene target for cAMPmediated transcription. Arg 1 catalyzes the conversion of arginine to urea and ornithine, a rate-limiting step for polyamine synthesis (Lange et al., 2004). Increased polyamines confer resistance of neurons to myelin inhibition and spermidine promotes optic nerve regeneration in vivo (Cai et al., 2002; Deng et al., 2009). Argl also protects motor neurons from trophic factor deprivationinduced apoptosis and cortical neurons from excitotoxicity by reducing arginine critical for nitric oxide (NO) synthesis (Dawson et al., 1991; Estévez et al., 2006). Collectively, these studies suggest that increasing arginase activity by elevating cAMP may be a promising therapeutic approach to promote CNS protection and regeneration.

An established strategy to increase cAMP in the CNS employs isoform-selective inhibition of phoshodiesterases (PDE) to suppress cAMP degradation. PDE inhibitors (e.g., rolipram) increase CAMP and promote regeneration in rodent models of SCI (Lu et al., 2004; Nikulina et al., 2004; Pearse et al., 2004) and prevent neuronal loss in stroke models (Kato et al., 1995; Block et al., 1997). However, their clinical use is limited by disabling nausea in some patients attributable to increases in cAMP in CNS emesis centers (Spina, 2008).

To identify drugs capable of protecting neurons and promoting axonal growth following injury, we focused on Arg1 for its ability to confer some of cAMP's salutary effects (Cai et al., 2002; Estévez et al., 2006). Indeed, we previously showed that cAMP induced Arg1 message and protein levels (Cai et al., 2002), and reasoned that an Arg1 promoter-reporter construct expressed in neuronal cells could be used to identify novel transcriptional activators of Arg1. As targeting transcription risks inadvertent toxicity, we screened a 2000 compound small molecule library that included clinically approved drugs and diverse natural compounds with a long record of safety in humans (Heemskerk et al., 2002).

\section{Materials and Methods}

All protocols involving animals were approved by the respective ILACUC committees of each institution and the NIH guidelines for use and care for laboratory animals. Animals used for the studies were obtained from Charles River Laboratories or Harlan.

Arg1 promoter screen. HT22 cells stably transfected with a luciferase reporter driven by $4.8 \mathrm{~kb}$ of DNA upstream of the Arg1 transcription start site were plated in 96 well culture plates for $24 \mathrm{~h}$ (Gray et al., 2005). Drug stocks $(1000 \times)$ in DMSO (MicroSource Spectrum library) were diluted in two steps (1:20 first, then 1:50 into final treatment media) to minimize precipitation, and then added to the plated cells. After incubation for $24 \mathrm{~h}$, luciferase activity was measured with a firefly luciferase assay (Promega) in an LMaxII luminometer (Molecular Devices). Luminescence readings were then normalized to well protein content measured by BCA protein assay (Pierce). As a secondary screen, HT22 cells were treated for $24 \mathrm{~h}$ with the compounds that increased Arg 1 reporter activity and then protein lysates were collected for Western blot analysis for Arg1 protein levels.

Neurite outgrowth assay on MAG-expressing $\mathrm{CHO}$ cells. The neurite outgrowth assay was performed on confluent monolayers of control $\mathrm{CHO}$ cells or myelin-associated glycoprotein (MAG)-expressing $\mathrm{CHO}$ cells in individual chambers of 8 well chamber slides (Nunc). Neurons were isolated from the DRGs or cerebellum of postnatal day 5 (P5)-P6 rats or from the cortices of embryonic day 18 (E18) rat embryos and cultured as previously described (Mukhopadhyay et al., 1994). For in vitro priming experiments, neurons were plated onto poly-L-lysinecoated culture dishes and incubated with the indicated drugs or vehicle overnight, trypsinized, and then resuspended in modified DMEM with
Sato supplement [200 nm progesterone, $224 \mathrm{~nm}$ selenium, $4 \mu \mathrm{g} / \mathrm{ml}$ insulin, $0.35 \mathrm{mg} / \mathrm{ml}$ bovine serum albumin (BSA), $0.4 \mu \mathrm{g} / \mathrm{ml} \mathrm{L}$-thyroxine, $0.34 \mu \mathrm{g} / \mathrm{ml}$ triiodothyronine]. Then, $1.5 \times 10^{4} \mathrm{DRG}, 5 \times 10^{4}$ cerebellar or $5 \times 10^{4}$ cortical neurons were plated upon the $\mathrm{CHO}$ monolayers for $18-48 \mathrm{~h}$, after which the cocultures were fixed with $4 \%$ paraformaldehyde (PFA) and permeabilized with ice-cold methanol for $2 \mathrm{~min}$. The cells were then blocked for 30 min in DMEM containing $10 \%$ fetal bovine serum (FBS) and incubated overnight with a rabbit polyclonal antibody against $\beta$ III-tubulin (1:1000, Tuj1, Covance) diluted in 5\% BSA/PBS. The cells were then washed three times with PBS and incubated with a biotinylated donkey anti-mouse IgG antibody (1:500, GE Healthcare) for $30 \mathrm{~min}$ at room temperature, washed three times with PBS, incubated with streptavidin-conjugated Texas Red (1:300, GE Healthcare) for 45 min, again washed three times with PBS, and mounted with Permafluor (Immunon). Images were captured with an epifluorescence microscope and analyzed using MetaMorph (Meta Imaging Software, Molecular Devices) image analysis software. The length of the longest neurite was determined for each neuron for the first 400-600 neurons encountered when scanning the slide in a systematic manner.

Turning response to focal sources of MAG. L4-6 DRGs from adult male Sprague Dawley rats were dissociated using collagenase and trypsin, plated at a low density on poly-L-lysine and laminin-coated surfaces, and cultured at $37^{\circ} \mathrm{C}$ with $5 \% \mathrm{CO}_{2}$ as previously described (Twiss et al., 2000). At the time of plating, neurons were cultured in the presence or absence of $80 \mu \mathrm{M}$ 5,6-dichlorobenzimidazole riboside (DRB), which inhibits RNA polymerase II. After $12 \mathrm{~h}$, cultures were treated with DMSO or daidzein $(10,20,30$ or $40 \mu \mathrm{M})$ for an additional $12 \mathrm{~h}$. For localized application of tropic stimuli to growth cones, $2.5 \mu \mathrm{g}$ of MAG-Fc (R\&D Systems) was covalently coupled to $4.5 \mu \mathrm{m}$ carboxylated polystyrene microspheres $\left(2.5 \times 10^{7}\right.$ microparticles $)$ per manufacturer's instructions (Polysciences). Fc-coated microspheres were used to test any nonspecific effects of coated microparticles. Ligand-immobilized microparticles were added to DRG cultures $24 \mathrm{~h}$ after plating. After allowing the particles to settle for $10 \mathrm{~min}$, DRGs were imaged using a Nikon Eclipse TS100 inverted microscope fitted with a QICAM 12-bit Fast1394 camera. Two hours after addition of the coated microparticles, DRGs were imaged again. The turning angle relative to the direction of the last $10 \mu \mathrm{m}$ segment of the axon shaft at the beginning of the experiment and the percentage of growth cone collapse were determined.

Western blot analysis. Cerebellar or cortical neurons plated on poly-Llysine or HT22 cells were treated with the indicated drugs, rinsed and then lysed with either RIPA or NP-40 buffer supplemented with phosphatase and proteinase inhibitors. Lysates were boiled for $5 \mathrm{~min}$ in denaturing buffer after which proteins were separated by SDS-PAGE through a $10 \%$ polyacrylamide gel and then transferred to nitrocellulose membranes. Blots were immunolabeled with antibodies against Arg1 (1:5000) (Esch et al., 1998), phosphorylated CREB (Ser-133, 1:1000, Millipore), or CREB (1:1000, Millipore) overnight at $4^{\circ} \mathrm{C}$, washed, and either incubated at room temperature for $1 \mathrm{~h}$ with HRP-conjugated antibodies against rabbit IgG (1:5000) or with IR-dye-conjugated antibodies against either rabbit or mouse IgG (1:15000, LI-COR Biosciences) and then rinsed three times. Blots incubated with HRP-conjugated secondary antibodies were visualized by chemiluminescence (ECL reagent, GE Healthcare) followed by exposure to radiology film. These blots were then stripped and reprobed with antibodies against $\beta$-actin (1:1000, Sigma). Blots incubated with IR-dyes were imaged with the Odessey IR quantitative Western blot system (LI-COR Biosciences).

Intrathecal and subcutaneous daidzein administration. Osmotic minipumps (Alzet model 1007D, $0.5 \mu \mathrm{l} / \mathrm{h}$ delivery rate) were filled with various concentrations of daidzein dissolved in DMSO or vehicle only (Sigma) and then incubated at $37^{\circ} \mathrm{C}$ for at least $6 \mathrm{~h}$ before implantation. For intrathecal delivery, P28-P30 Fisher rats were anesthetized with isoflurane and the spinal cord at L5 was exposed by laminectomy. An incision was made in the dura mater and the tip of a cannula attached to the minipump was guided into the subdural space along the surface of the spinal cord and secured by a suture thread. For subcutaneous delivery, minipumps were inserted per manufacturer's protocols. Briefly, equilibrated osmotic minipumps were implanted subcutaneously through a midscapular incision in deeply anesthetized rats. The filled minipumps 
were inserted delivery portal first, after which the surgical opening was closed with wound clips. Animals were killed $1 \mathrm{~d}$ after surgery and the L4-L5 DRG neurons were isolated and plated upon CHO monolayers for the neurite outgrowth assay as described above.

Optic nerve crush and intraocular and subcutaneous daidzein administration. Fisher rats (250-300 g) were deeply anesthetized with isoflurane and immobilized in a stereotaxic apparatus. Optic nerves were surgically exposed and the dural sheath surrounding the optic nerve was carefully incised longitudinally. The optic nerve was then crushed $2 \mathrm{~mm}$ from the orbit with a \#5 jeweler's forceps for $10 \mathrm{~s}$. The surgical site was sutured closed and the preservation of the central retinal artery was verified by direct fundoscopic examination for signs of ischemic damage. Animals showing ischemic damage were excluded from the study. Immediately following the nerve crush, animals were either injected intraocularly with $10 \mu \mathrm{l}$ of the indicated concentration of daidzein or DMSO with a pulledglass pipette using a nano-injector (WPI) or implanted subcutaneously with an osmotic minipump loaded with daidzein as described above. Animals were observed for postoperative recovery and were housed individually with ad libitum access to food and water. Two weeks later, animals were deeply anesthetized with ketamine/xylazine $(100 \mathrm{mg} / \mathrm{kg}$ and $10 \mathrm{mg} / \mathrm{kg}$ respectively) and transcardially perfused with $200 \mathrm{ml}$ of heparinized saline (1000 U/L) followed by $300 \mathrm{ml}$ of $4 \%$ PFA. The optic nerve was removed, postfixed overnight in $4 \% \mathrm{PFA}$, and cryoprotected in $30 \%$ sucrose in Tris-buffered saline (TBS). Additionally, the lens from each eye was examined for injury. Animals exhibiting lens injuries were excluded from the study.

Immunohistochemistry and image analysis. Optic nerves were embedded in freezing medium and $20 \mu \mathrm{m}$ serial sections were cut and mounted onto glass slides. The sections were washed four times with TBS, blocked with TBS $+0.2 \%$ Triton X-100 $+5 \%$ normal goat serum for $1 \mathrm{~h}$, and then incubated overnight at $4^{\circ} \mathrm{C}$ with a sheep antibody against growthassociated protein-43 (GAP-43; 1:100, a generous gift from Dr. L. Benowitz, Harvard School of Medicine, Boston, MA). The sections were then washed four times with TBS and incubated with a FITC-conjugated goat antibody against sheep IgG (1:500) for $1 \mathrm{~h}$ at room temperature. Sections were again washed and mounted with an aqueous mounting medium before imaging. A high resolution image montage was automatically acquired from a Nikon Eclipse 90 i fluorescent microscope at $20 \times$ magnification and assembled using MetaMorph acquisition software. The distance traversed by the three longest GAP-43-positive axons relative to the distal edge of the crush injury site was measured from three sections for each animal.

Neuron survival assays. Spinal motor neurons were purified from E14.5 rat embryos as previously described. Briefly, ventral spinal cord tissue was dissected and dissociated, and enriched for motor neurons by centrifugation through an Optiprep gradient. Motor neurons were further purified by magnetic bead cell separation (Miltenyi Biotec) for cells expressing P75 ${ }^{\mathrm{NTR}}$ receptor, which is expressed in motor neurons at this developmental age. The resulting cultures were $96 \%$ motor neurons as assessed by Islet $1 / 2$ immunocytochemistry. The cells were plated in 96 well plates in Neurobasal media + B27 supplement (Invitrogen) in the presence or absence of brain-derived neurotrophic factor (BDNF, $1 \mathrm{ng} /$ $\mathrm{ml})$. Daidzein $(20 \mu \mathrm{M})$ or DMSO was added at the time of plating. Motor neurons were assayed for viability $24 \mathrm{~h}$ later using calcien-AM (Invitrogen) uptake followed by unbiased cell counting using a Flash Cytometer (Trophos). Cortical neurons were cultured from E17 rat embryos as previously described. After $1 \mathrm{~d}$ in culture, neurons were cotreated with daidzein and homocysteic acid (HCA, $5 \mathrm{~mm}$ ), which prevents the uptake of cystine and causes glutathione depletion leading to apoptosis in embryonic neurons. The cultures were assayed for viability $24 \mathrm{~h}$ later with an MTT assay (Promega) according to the manufacturer's protocols.

cAMP measurements. Intracellular cAMP was measured with a competitive colorimetric immunoassay kit according to the manufacturer's protocols (Millipore). In this assay, alkaline phosphatase-conjugated cAMP competes with cAMP from cell lysates for binding to a cAMPspecific antibody. The conjugated cAMP is then detected using an alkaline phosphatase substrate. Primary cortical neurons $[1$ day in vitro (DIV)] were treated overnight with daidzein and then assayed for cAMP. The alkaline phosphatase activity of each sample was measured in a Spec-
traMax spectrophotometer (Molecular Devices) and compared with a cAMP standard curve for quantitation.

Estrogen promoter activity. Estrogen promoter activity was assessed using a firefly luciferase reporter gene under the control of three copies of the vitellogenin estrogen response element (ERE) (Hall and McDonnell, 1999). Naive HT22 cells were transiently transfected with the ERE reporter using Lipofectamine 2000 (Invitrogen) and then treated with $17 \beta$ estradiol with or without fulvestrant, an estrogen receptor antagonist. After $24 \mathrm{~h}$, the cells were lysed and assayed for luciferase activity as above.

Statistical analysis. All data were expressed as mean \pm SEM and were plotted using GraphPad Prism software. The statistical tests used for analysis are indicated in the figure legends. Statistical significance was taken as $p<0.05$.

\section{Results}

\section{Biological screening of drug library}

We generated a pool of stable immortalized hippocampal cell clones [HT22ap (arginase promoter)] that express a firefly luciferase reporter driven by a large portion of the proximal 5' noncoding region $(4.8 \mathrm{~kb})$ of the murine Arg1 gene. To evaluate the validity of this tool for analyzing arginase transcription in a neuronal context, we first examined the effect of dibutyryl-cAMP (db-cAMP), a membrane-permeable and nonhydrolyzable form of the second messenger cAMP, that was previously shown to induce Arg1 transcription in primary cortical and DRG neurons (Cai et al., 2002). As expected, we found robust and reproducible induction of Arg1 mRNA in HT22 cells. This induction of message was accurately reported by our HT22ap line. Induction of Arg1 by db-cAMP was attributable to increases in cAMP (and not butyrate) as it effects were mimicked by the PDE4 inhibitor rolipram. Moreover, actinomycin $\mathrm{D}$, a canonical inhibitor of transcription, completely abrogated the cAMP-mediated increase in Arg1 message and luciferase activity, demonstrating that the cell line and reporter system were ideal tools to identify compounds to regulate Arg1 in neurons (data not shown).

To assess the induction of Arg1 in HT22ap line, we screened a commercially available small molecule library containing 2000 Food and Drug Administration (FDA)-approved drugs, natural products, and other biologically active compounds (MicroSource Spectrum library) using a luminometer to detect luciferase production (Fig. 1A,B). From this initial screen, we identified 40 compounds that induced luciferase activity by at least twofold, an amount similar to our positive control, $1 \mathrm{~mm}$ db-cAMP (Fig. 1 B). "Hits" from the initial screen (performed at $10 \mu \mathrm{M})$ such as daidzein exhibited a concentration-dependent induction of activity at the Arg1 promoter (Fig. 1C). To confirm that changes in reporter activity reflected increases in protein, we performed immunoblot analysis on native HT22 cells using an antibody specific for Arg1 (Fig. 1D) (Estévez et al., 2006). Of the small molecules that increased reporter activity, only 10 increased Arg1 protein levels. Interestingly, many inducers of Arg1 protein were isoflavones or intermediates/precursors to flavanoid biosynthetic pathways (Fig. $1 E$ ). However, not all isoflavones were true hits. For example, daidzein induced Arg1 levels whereas genistein, which differs from daidzein by only one hydroxyl group, was ineffective (Fig. $1 D, E$; lane 13 = daidzein, lane 20 = genistein). Additionally, while many flavones increased reporter activity, only methoxyvone was positive in the secondary screen (Fig. $1 D, E$; lane $12=4,7$-dimethyoxyflavone, lane $31=$ methoxyvone, lane 32 = ginkgetin).

From the initial 40, we selected 12 compounds, three true hits and nine false positives, to test in a blind screen for the ability to overcome neurite outgrowth inhibition by MAG. Using a model previously developed in one of our labs, cerebellar 
granule neurons (CGN) were plated upon a monolayer of control $\mathrm{CHO}$ cells or upon $\mathrm{CHO}$ cells expressing MAG (MAG-CHO) (Mukhopadhyay et al., 1994). Plating neurons on MAG-CHO cells significantly inhibits neurite outgrowth. When added concurrently with neurons at plating, no effect on MAG's inhibition of neurite outgrowth was seen with any of these $12 \mathrm{com}$ pounds (at 5, 10, and $30 \mu \mathrm{M}$ ). In contrast, db-cAMP, a positive control, completely overcame MAG inhibition as previously reported (data not shown). Interestingly, neurotrophins added at plating are also unable to promote neurite outgrowth on MAG substrates; however neurons "primed" with neurotrophins before plating are able to overcome MAG inhibition (Cai et al., 1999). Using this priming protocol, we first plated CGNs on poly-llysine-coated plates in the presence of the same amounts of each compound overnight. The treated neurons were then trypsinized, transferred onto monolayers of control or MAG-expressing $\mathrm{CHO}$ cells, and allowed to grow overnight before fixation and immunolabeling for $\beta$ III-tubulin. Under these conditions, only one compound was able to completely overcome MAG inhibition, while one other promoted modest neurite outgrowth (Fig. 2A). Importantly, these two compounds, daidzein and methoxyvone, were among the three true hits identified in the Arg1 screen, validating our strategy of using Arg 1 induction as a biological marker to screen for molecules that overcome myelin-associated axonal growth inhibition.

\section{Daidzein is effective in many types of neurons and requires arginase activity to overcome MAG inhibition}

As daidzein was the most effective compound in overcoming MAG inhibition, we further characterized daidzein in vitro to assess its potential as a therapeutic agent (Fig. 2A). Using increasing concentrations of daidzein, we found that priming with $20 \mu \mathrm{M}$ was most effective in promoting neurite outgrowth of CGNs on MAG-CHO cells (data not shown). This dose response for daidzein was reproduced in three separate laboratories in three different institutions, providing internal replication for the initial observations (Fig. $2 \mathrm{H}, \mathrm{I}$ and data not shown). At $20 \mu \mathrm{M}$ daidzein, $\operatorname{Arg} 1$ protein levels were increased threefold following overnight incubation, which is similar in magnitude to treatment with $2 \mathrm{~mm}$ db-cAMP, which we have previously shown to increase Arg1 levels (Fig. 2B). As arginase activity is required for cAMP and neurotrophins to overcome MAG inhibition, we used a specific inhibitor of arginase activity, $N^{\omega}$-hydroxy-nor-L-arginine (nor-NOHA), and found that the daidzein-mediated increase in axonal outgrowth methoxyvone, and derrustone.

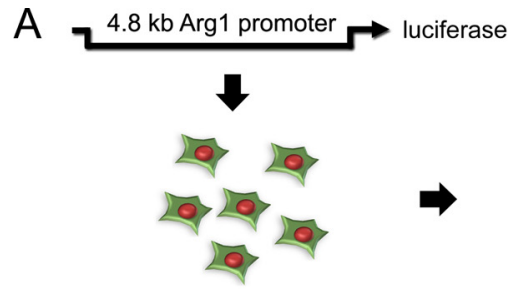

B

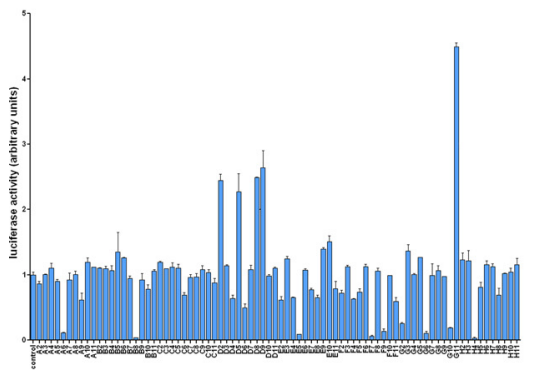

daidzein (13)

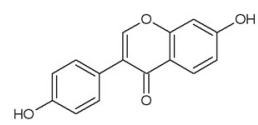

genistein (20)
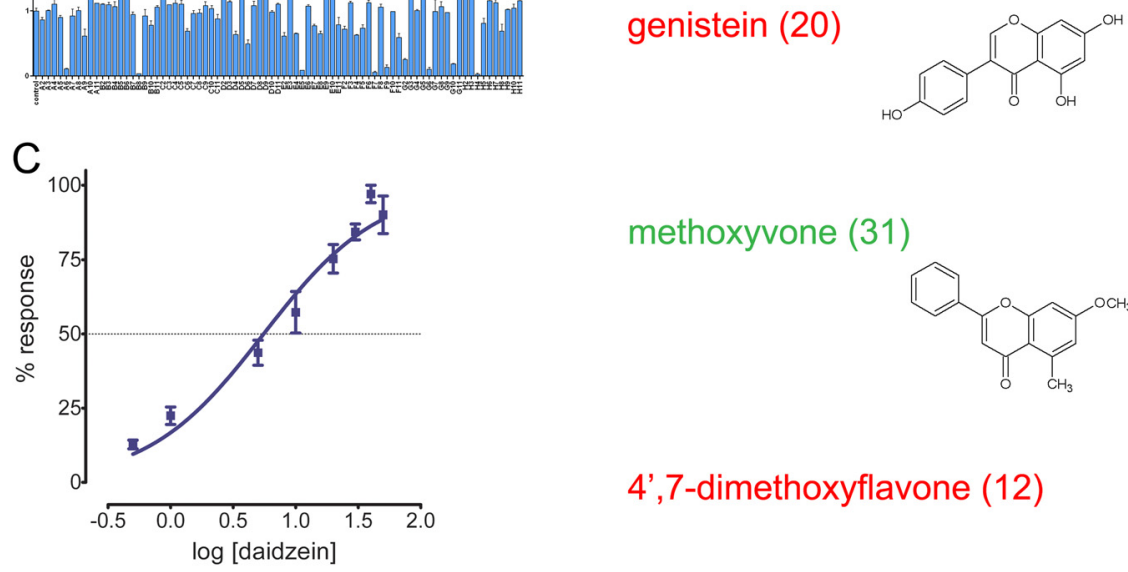

methoxyvone (31)

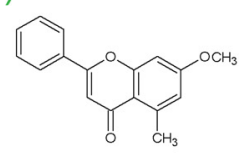

4',7-dimethoxyflavone (12)
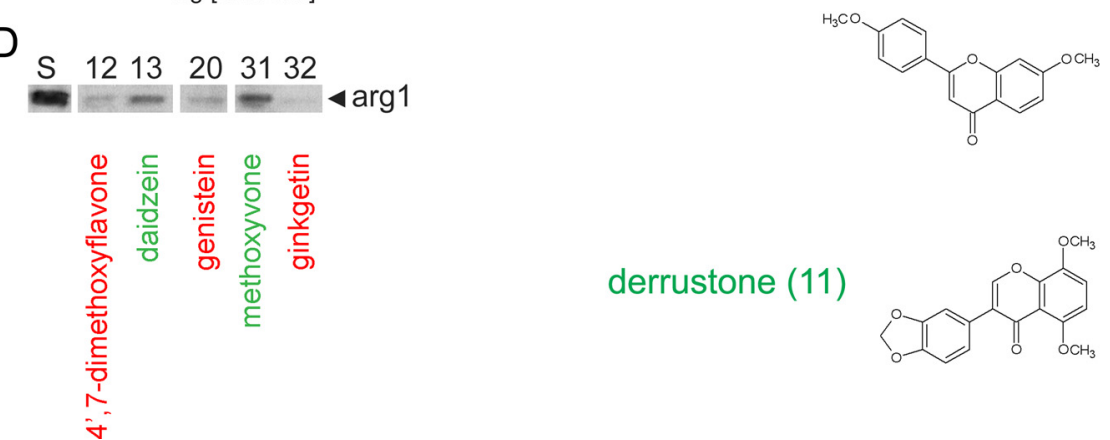

derrustone (11)

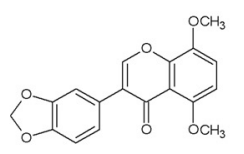

Figure 1. Biological screen for inducers of Arg1. A drug library containing 2000 FDA-approved drugs, natural products, and other biologically active small molecules was screened for inducers of Arg1 using a luciferase reporter driven by $4.8 \mathrm{~kb}$ of the Arg1 promoter in HT22 cells. $\boldsymbol{A}$, Schematic representation of the screening procedure. $\boldsymbol{B}$, Representative plot of data from one 96 well plate. Molecules that induced Arg1 reporter activity by at least twofold were selected as hits. C, Daidzein concentration —response curve $(\mu \mathrm{m})$ for activity at the Arg1 promoter. D, Protein lysates from cells treated with indentified compounds were immunoblotted for Arg1 protein levels as a secondary screen. Not all compounds identified in the reporter screen increased Arg1 protein levels. The lanes are: S, recombinant Arg1 protein; 12, 3,7-dimethyloxyflavone; 13, daidzein; 20, genistein; 31, methoxyvone; and 32, ginkgetin. $E$, Of the 10 compounds identified and confirmed in the secondary screen, many were flavanoids including daidzein,

on MAG CHO cells was completely inhibited by coincubation with the antagonist (Fig. 2C-G) (Cai et al., 2002). Importantly, nor-NOHA alone did not inhibit neurite outgrowth in CGNs plated on control CHO cells (Fig. $2 C, G$ ). Together, these data show that daidzein upregulates Arg1 protein levels and that arginase activity is necessary for overcoming MAG-mediated inhibition of axonal outgrowth in CGNs.

Recent studies have highlighted the cell type-specific roles that Nogo receptors play in inducing glial inhibition (Mehta et al., 2007; Venkatesh et al., 2007). In contrast, cAMP appears to 

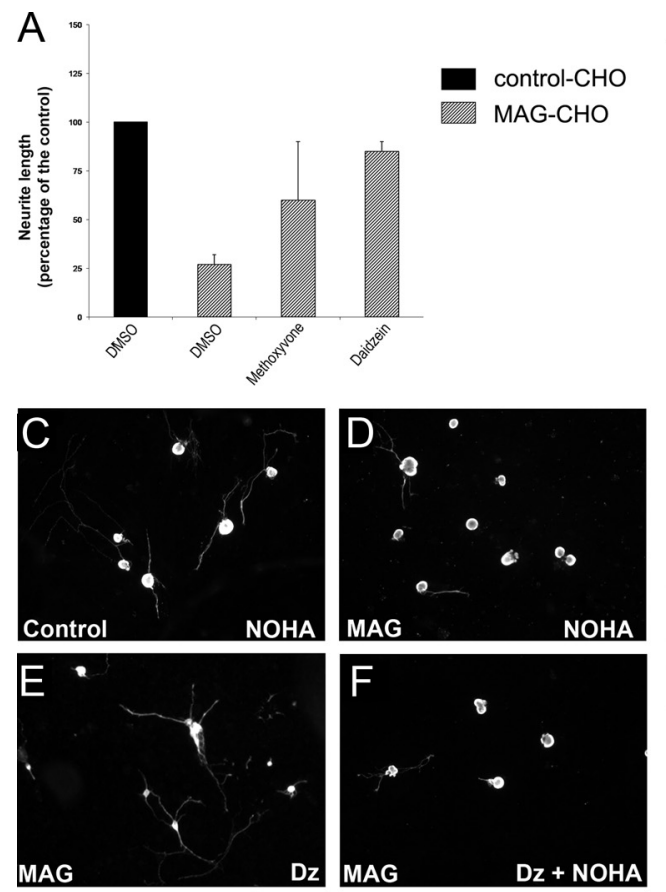

B

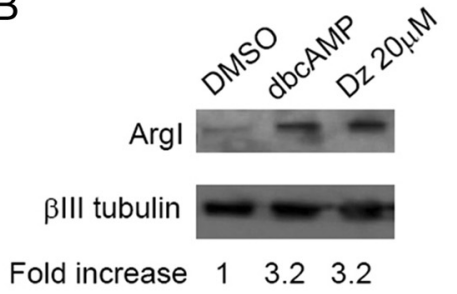

G $\quad$ control-CHO
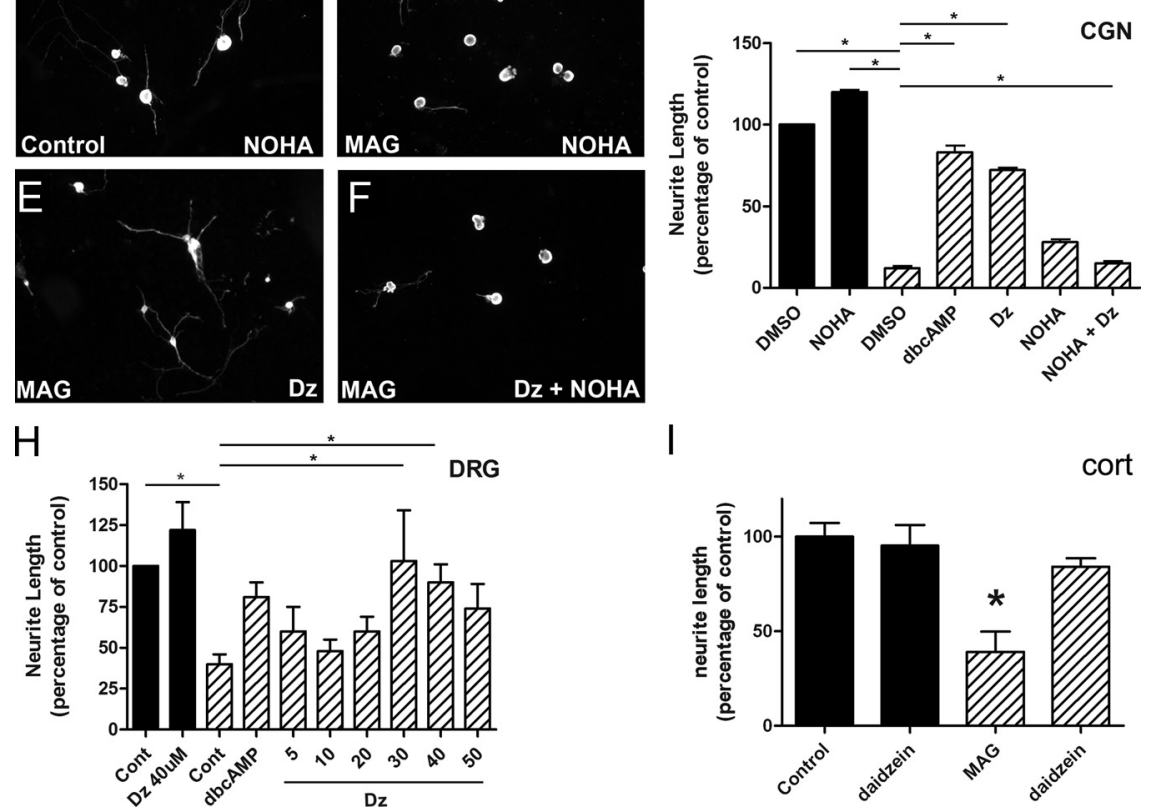

1

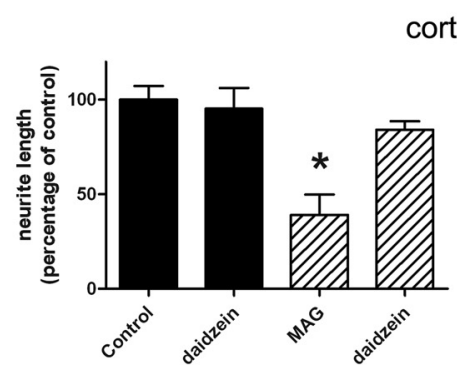

Figure 2. Daidzein overcomes MAG inhibition through inducing Arg1 activity. $\boldsymbol{A}$, In a blind screen of 12 compounds ( 3 true hits and 9 false positives), only (GNs primed with methoxyvone and daidzein overcame MAG-mediated inhibition of neurite outgrowth. Daidzein was further characterized due to its superior efficacy. $\boldsymbol{B}, \mathrm{Arg} 1 \mathrm{protein}$ levels are increased by daidzein $(20 \mu \mathrm{M})$ to the same extent as db-cAMP (2 mm). Arginase activity is required for daidzein-mediated axonal outgrowth on MAG-CHO cells $(\mathbf{C}-\mathbf{G})$. CGNs grown on control CHO cells incubated with NOHA (C) exhibit normal neurite outgrowth, whereas CGNs plated on MAG-CHO cells (D) fail to extend neurites. $\boldsymbol{E}$, Priming CGNs with daidzein $(20 \mu \mathrm{m})$ for $24 \mathrm{~h}$ overcomes MAG inhibition, which was reversed by the inclusion of nor-NOHA (10 $\mu \mathrm{m})$, an inhibitor of arginase activity. G-I, Neurite outgrowth was quantitated in neurons primed with daidzein and then plated on control $\mathrm{CHO}$ cells (closed bars), or in neurons grown on MAG-CHO cells (diagonal bars). P0 CGNs $(\boldsymbol{G})$, P5-P6DRG neurons $(\boldsymbol{H})$, or E18 cortical neurons $(\boldsymbol{I})$ treated with daidzein were resistant to inhibition by MAG. These data were repeated in at least three independent experiments, ${ }^{*} p<0.001$, one-way ANOVA with Tukey's post hoc test.

be effective in diverse cell types in activating pathways to overcome glial inhibition (Cai et al., 1999). To assess whether daidzein, like cAMP, was effective in overcoming MAG inhibition in diverse brain regions, we used DRG neurons (which have a CNS axon that ascends in the spinal cord) and embryonic cortical neurons. Both DRG (Fig. $2 H$ ) and cortical neurons (Fig. $2 I$ ) primed with daidzein and subsequently plated upon MAG-CHO cells were resistant to inhibition of neurite outgrowth by MAG. Unlike CGNs, the maximal effect of daidzein occurred from 30 to $40 \mu \mathrm{M}$ in DRG neurons and at $10 \mu \mathrm{M}$ in cortical neurons, suggesting that the sensitivity to daidzein depends on neuronal cell type. Daidzein also rendered growth cones of adult DRG neurons resistant to the collapse and repulsion induced by focal sources of MAG (Fig. $3 A, B, E$ ). As predicted, daidzein's effects are completely abrogated in cultures exposed to DRB (Fig. 3C-E), which inhibits $>90 \%$ of new RNA synthesis in these adult DRG cultures (Willis et al., 2007). Importantly, these data confirm that daid- zein acts in a transcription-dependent manner to overcome MAG inhibition in different types of primary neurons.

Daidzein is biologically active in vivo and crosses the blood-brain barrier In dissociated cultures, neurons have direct access to drugs applied to the culture medium. To determine whether daidzein reaches its effectors in vivo, we administered daidzein intrathecally to see whether local delivery of the drug was sufficient to prime DRG neurons to overcome MAG inhibition. Daidzein at 20 or $40 \mu \mathrm{M}$ was continuously administered through osmotic minipumps for $24 \mathrm{~h}$. The animals were then killed, DRGs removed, dissociated, and directly plated upon control $\mathrm{CHO}$ or MAG-CHO monolayers. DRG neurons from animals receiving vehicle (DMSO) exhibited significant neurite outgrowth on control CHO cells, whereas this neurite outgrowth was significantly blunted on MAG-CHO cells (Fig. 4A,B). Most important, DRG neurons from animals receiving $40 \mu \mathrm{M}$ daidzein overcame inhibition by MAG (Fig. $4 C, G$ ) while the $20 \mu \mathrm{M}$ dose had no effect (data not shown), indicating that daidzein delivered locally in vivo is biologically active at the concentrations effective in vitro.

While intrathecal delivery of daidzein was effective, this route of administration is inconvenient, invasive, and has potential for serious complications, particularly in the context of an injured CNS. An ideal drug would be effective following peripheral administration, either into the systemic circulation (i.e., subcutaneously) or orally. For this to be effective, the drug must also cross the blood-brain barrier to reach its site of action. To test this, we implanted daidzein-containing minipumps subcutaneously for $24 \mathrm{~h}$ before removing DRG neurons for plating on MAG-CHO cells. As the drug must travel through the systemic circulation when administered by this route, we reasoned that much higher concentrations of daidzein were required than in vitro or for local delivery and started with millimolar doses of the drug released at $0.5 \mu \mathrm{l} / \mathrm{h}$. We found that concentrations of $2 \mathrm{~mm}$ and higher were effective in overcoming MAG inhibition, with the maximum effect occurring at $12 \mathrm{~mm}$ (Fig. $4 F, H$ ). Together, our results strongly suggest that daidzein is biologically active in vivo, enters the CNS when given peripherally, and may be a viable therapeutic agent to promote axonal regeneration following CNS injury.

\section{Daidzein promotes optic nerve regeneration following crush injury}

To determine whether the beneficial effects of daidzein in the MAG inhibition model translated to improved axonal outgrowth following injury in vivo, we tested daidzein in an optic nerve crush injury and regeneration model. The axons of the retinal ganglion 
neurons, which reside within the CNS, were mechanically injured $\sim 2 \mathrm{~mm}$ behind the eye. Immediately following the crush injury procedure, daidzein (100 $\mu \mathrm{M}-4 \mathrm{mM})$ was injected intraocularly, taking care to not damage the lens. Animals with damaged lenses were not included in the analysis as lens lesions may also promote recovery (Leon et al., 2000; Fischer et al., 2001). After 2 weeks, the animals were killed, the injured optic nerves removed, sectioned, and immunolabeled for GAP43, a marker for injured/regenerating axons. In vehicle-treated animals, GAP43 labeling showed that few injured axons grew beyond the lesion site (Fig. 5A; asterisk denotes site of injury, arrows denote regenerated axons). In contrast, axons were able to regenerate in animals receiving daidzein for significant distances beyond the distal margin of the injury (Fig. 5B; DMSO: $436.6 \pm 133.8 \mu \mathrm{m}$ vs daidzein: $2035.2 \pm 295.8 \mu \mathrm{m}, p<0.05$, Student's $t$ test).

As intraocular delivery exposes the retinal ganglion cell bodies directly to daidzein, we wanted to know whether systemic delivery of daidzein also afforded similar regenerative enhancement. We used a postinjury paradigm where daidzein was delivered subcutaneously with osmotic minipumps for the 2-week recovery period following the optic nerve crush injury. In agreement with local delivery, animals receiving subcutaneous daidzein exhibited longer regenerated axons, extending up to $7 \mathrm{~mm}$ beyond the injury site (Fig. 5C), confirming that daidzein was effective in promoting axonal regeneration when administered subcutaneously.

\section{Daidzein does not affect cAMP levels or} CREB phosphorylation

The elevation of neuronal cAMP overcomes MAG inhibition by activating PKA, leading to phosphorylation of CREB at serine 133, and subsequent recruitment of the coactivator $\mathrm{CBP} / \mathrm{p} 300$ to transactivate a host of genes, including Arg1 (Cai et al., 2002). To determine whether daidzein affected the CAMP-PKA-CREB pathway, we measured cAMP levels in cortical neurons following daidzein treatment and found that in contrast to db-cAMP, treatment with daidzein for 2 or $24 \mathrm{~h}$ did not increase intracellular cAMP levels (data not shown and Fig. $6 A$, respectively). To further rule out CREB-mediated induction of Arg1 expression, we treated cortical neurons with db-cAMP (5 mM) or daidzein (1-40 $\mu \mathrm{M}$; only $20 \mu \mathrm{M}$ is shown), collected protein lysates at $0,2,4,6$, and $24 \mathrm{~h}$, and immunoblotted using an antibody that
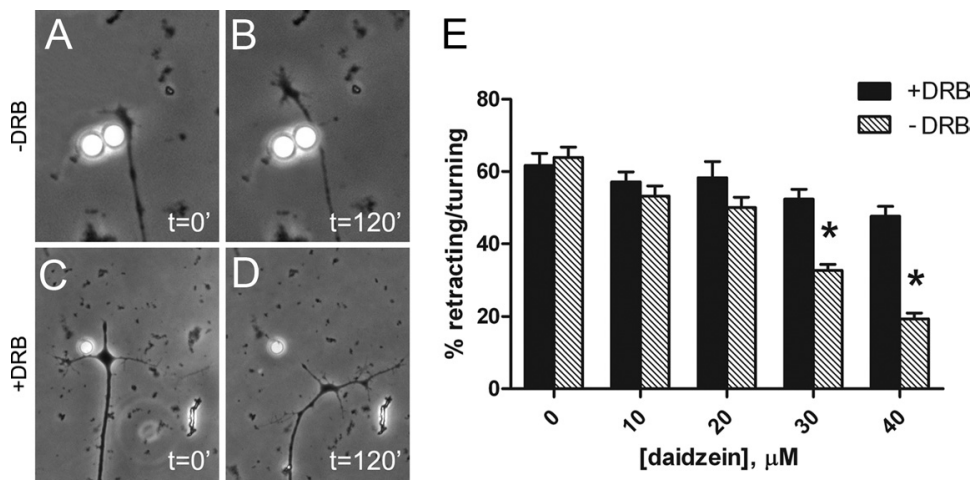

Figure 3. Daidzein prevents growth cone collapse and repulsion from MAG-coated microparticles. Adult DRGs were grown for $12 \mathrm{~h}$ in the presence or absence of the RNA polymerase II inhibitor DRB and then treated with vehicle (DMSO) or increasing concentrations of daidzein for an additional $12 \mathrm{~h}$. $A$, A representative micrograph of a terminal axon and growth cone, cultured in the absence of DRB, stimulated with a MAG-Fc-coated microparticle. $\boldsymbol{B}$, In the absence of DRB, pretreatment with $40 \mu \mathrm{m}$ daidzein prevents the axon from responding to the MAG stimulus and the axon continues to grow along its trajectory, growing past the microparticle within $120 \mathrm{~min}$. C, A representative micrograph of a terminal axon and growth cone cultured in the presence of $80 \mu \mathrm{m}$ DRB, stimulated with a MAG-Fc-coated microparticle. D, Pretreatment with DRB blocks the daidzein effect and the axon turns away from the MAG stimulus. $E$, Quantification of axon turning/retraction in response to microparticles loaded with MAG-FC. Pretreatment with daidzein at 30 or $40 \mu \mathrm{m}$ attenuates the MAG response. This attenuation is blocked by addition of the transcriptional inhibitor DRB. These data were repeated in three independent experiments, with at least 20 axons imaged per experiment, ${ }^{*} p<0.001$, two-way ANOVA followed by Bonferroni's post hoc test.
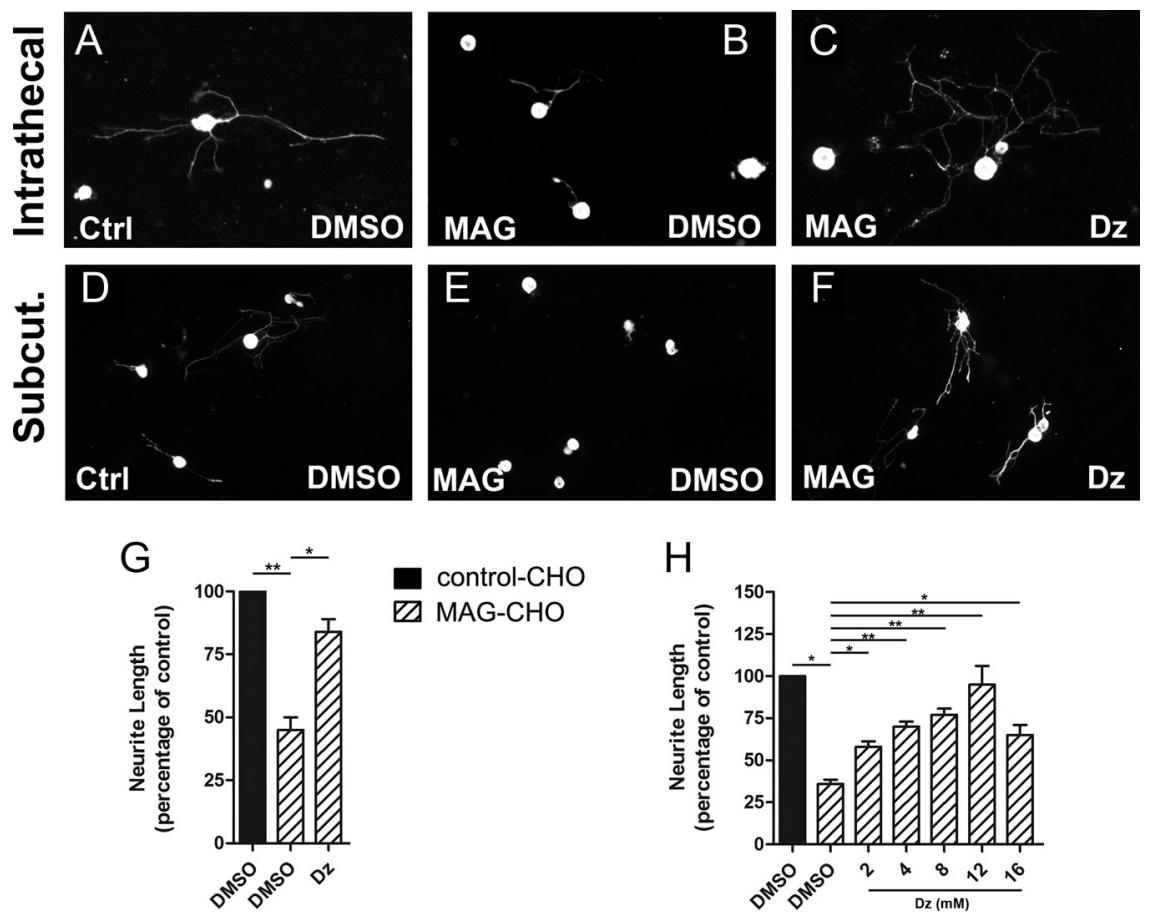

Figure 4. Daidzein primes in DRG neurons when administered in vivo and crosses the blood-brain barrier. Daidzein was administered as indicated, after which DRG neurons were plated on control or MAG-CHO cells for $24 \mathrm{~h}$, and assayed for neurite outgrowth by $\beta$ III-tubulin fluorescence immunolabeling. $\boldsymbol{A}-\boldsymbol{C}$, To determine whether in vivo administration of daidzein primes DRG neurons to overcome MAG inhibition, we infused P28 -P30 rats with daidzein intrathecally using osmotic minipumps for $24 \mathrm{~h}$ before removing DRG neurons for culture on $\mathrm{CHO}$ cells. $\boldsymbol{A}$, Neurons from vehicle-infused animals on control $\mathrm{CHO}$ cells; $\boldsymbol{B}$, neurons from vehicle-infused animals on MAG-CHO cells; $\boldsymbol{C}$, neurons from animals infused with $40 \mu$ m daidzein on MAG-CHO cells. $\boldsymbol{D}-\boldsymbol{F}$, To test whether daidzein crossed the blood-brain barrier and was effective in priming DRG neurons when given peripherally, daidzein was administered to P28 -P30 rats through subcutaneously implanted osmotic minipumps for $24 \mathrm{~h}$ before removal of DRG neurons. $\boldsymbol{D}$, Neurons from vehicle-treated animas on control $\mathrm{CHO}$ cells; $\boldsymbol{E}$, neurons from vehicle-treated animals on MAG-CHO cells; $\boldsymbol{F}$, neurons from animals treated with $8 \mathrm{~mm}$ daidzein on MAG-CHO cells. $\boldsymbol{G}, \boldsymbol{H}$, Analysis of neurite lengths from intrathecal infusion $(\boldsymbol{G})$ and subcutaneous administration $(\boldsymbol{H})$ show that daidzein primes DRG neurons to overcome MAG inhibition when given in vivo (black bars, neurons plated on control CHO cells; diagonal bars, neurons plated on MAG-CHO cells). The efficacy of subcutaneous administration suggests that daidzein crosses the blood-brain barrier. Data from in vivo priming experiment represent two animals per condition from two independent experiments, ${ }^{*} p<0.01$, one-way ANOVA with Tukey's post hoc test. 

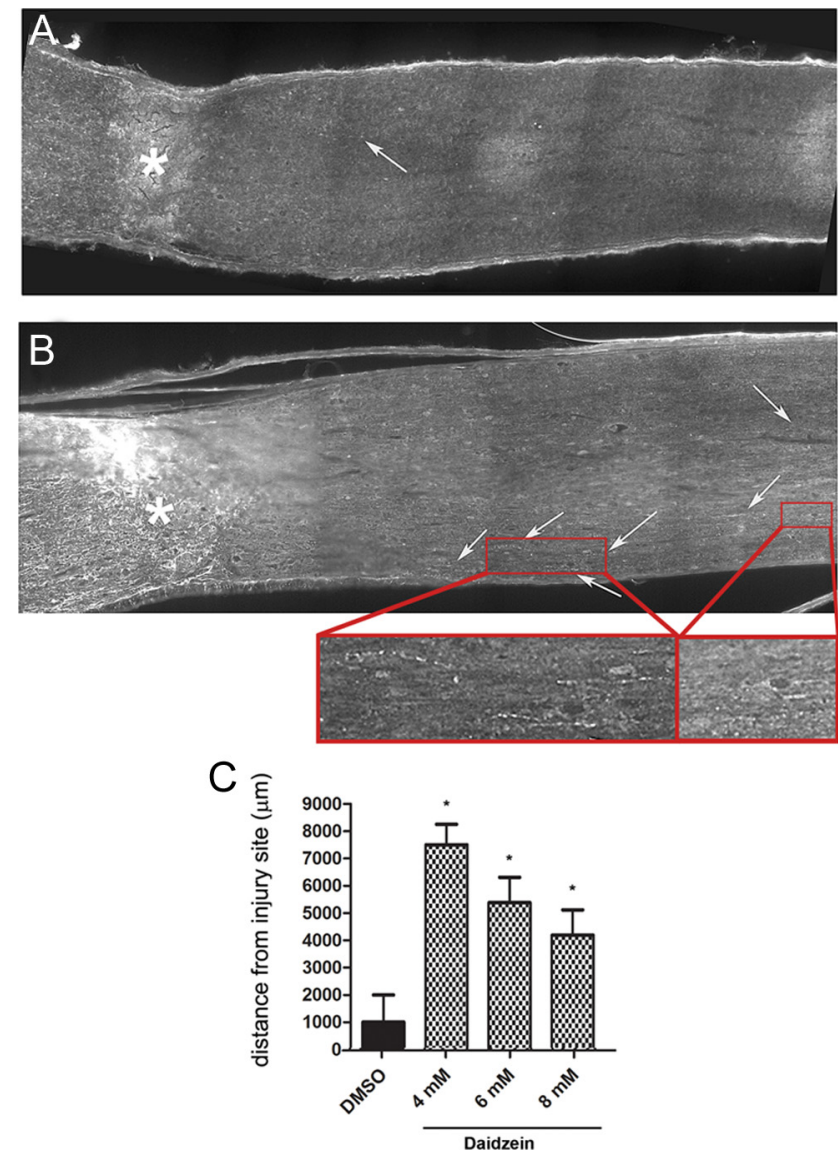

Figure 5. Daidzein promotes optic nerve regeneration in vivo following an optic nerve crush injury. The optic nerves of P28-P30 rats were crushed approximate $2 \mathrm{~mm}$ behind the eye and allowed to recover for 2 weeks. Daidzein was administered intraocularly immediately following the injury, taking care to not damage the lens, or subcutaneously for the 2-week recovery period. Optic nerves were removed, sectioned, and immunolabeled for GAP43-positive axons (arrows). A, GAP43-labeled injured optic nerve from an animal that received intraocular vehicle. $B$, An injured optic nerve from an animal that received intraocular daidzein. Note the increase in GAP43-positive axons (arrows) growing beyond the lesion site (asterisk) in daidzein-treated animals. $C$, The distance of regeneration beyond the injury site of the three longest axons was measured in each section from animals treated subcutaneously with vehicle or daidzein. Importantly, these data show that daidzein is effective in an in vivo injury model when given peripherally and after the injury. Data represent mean \pm SEM for 5 animals per group, ${ }^{*} p<0.05$, one-way ANOVA with Tukey's post hoc test.

recognizes CREB phosphorylation at serine- 133. With db-cAMP, maximal phosphorylated CREB (pCREB) levels were reached by $2 \mathrm{~h}$ and gradually declined over $24 \mathrm{~h}$. As expected, inhibiting protein kinase A (PKA) with the pharmacological inhibitor H89 $(10 \mu \mathrm{M})$ prevented the cAMP-induced phosphorylation of CREB (Fig. $6 B$ ). In contrast, daidzein treatment did not elevate pCREB levels at any of the time points measured (Fig. 6B). Thus, we conclude that daidzein is unlikely to be increasing cAMP levels or activating PKA to induce Argl expression.

\section{Estrogen receptor activity is necessary but insufficient to activate the Arg1 promoter}

Daidzein has been reported to bind and activate estrogen receptors (Choi et al., 2008). To determine whether estrogen receptors play a role in Arg1 induction, we treated our HT22 Arg1 reporter cell line with daidzein $(20 \mu \mathrm{M})$ and/or fulvestrant, an estrogen receptor-specific antagonist. Fulvestrant inhibited both basal and daidzein-induced Arg1 promoter activity in a dose-dependent manner (Fig. 6C). We also found similar results with tamoxifen, another estrogen receptor antagonist, although only at high concentrations (data not shown). We next asked whether activation of estrogen receptors alone was sufficient to induce Arg1 promoter activity. Surprisingly, $17 \beta$-estradiol was unable to mimic the effects of daidzein as Arg1 promoter activity remained unchanged (Fig. 6D). Parallel experiments confirmed that the $17 \beta$ estradiol we used was active, as it could activate luciferase expression driven by the ERE in HT22 cells (Fig. 6E).

\section{Daidzein protects neurons in oxidative models of cell death} Our model posits that agents that can simultaneously recapitulate established benefits of neuroprotective agents and foster regeneration will be superior in promoting recovery after brain and spinal cord injury compared with established neuroprotective compounds. Prior studies have reported neuroprotective roles for daidzein, but the mechanisms for this effect are obscure. As forced expression of Arg1 is sufficient to protect motor neurons from trophic factor deprivation-mediated cell death (by inhibiting NO generation and/or generating polyamines), we assessed whether daidzein could induce Arg1 in motor neurons (Estévez et al., 2006). Using spinal cord motor neurons purified from E14.5 rat embryos, we verified that daidzein could induce Arg1 protein expression (Fig. 7A). Motor neurons grown in culture require the addition of trophic factors such as BDNF. When plated in the absence of trophic factors, $\sim 40 \%$ of neurons undergo NO/peroxynitrite-dependent apoptosis, which, as expected, was completely attenuated by daidzein $(20 \mu \mathrm{M})$ (Fig. $7 B)$. As other oxidants also participate in acute CNS injury, we tested daidzein in an in vitro model of oxidative injury in cortical neurons. Cell death in this model (embryonic neurons 1-2 DIV) does not involve activation of cell surface glutamate receptors (these receptors are expressed later in vitro (10-21 DIV)) but rather accrues from the ability of glutamate or its analogs (HCA) to block uptake of the amino acid cystine into neurons. Cellular cystine deprivation leads to depletion of the versatile antioxidant glutathione and $\mathrm{NO}$ independent oxidative death (Esch et al., 1998; Ratan et al., 2002). Treatment of cortical neurons with HCA for $24 \mathrm{~h}$ kills $\sim 70 \%$ of neurons and this oxidative glutamate toxicity was significantly inhibited by daidzein (Fig. 7C). Together, these data suggest that daidzein could capture the prior benefits of neuroprotective agents with the additional benefit of being able to enhance the ability of preserved neurons to overcome glial inhibition.

\section{Discussion}

Agents that increase cAMP such as PDE inhibitors have captured the attention of those interested in acute and chronic neurological disorders because of their dual ability to foster survival and enhance regeneration (Gong et al., 2004; DeMarch et al., 2007; Sasaki et al., 2007; DeMarch et al., 2008; Yang et al., 2008). While candidate PDE4 inhibitors are moving toward testing in humans afflicted with CNS injury, side effects such as nausea may limit their use (Davis et al., 2009). Prior studies by our groups and others have attributed some of the prosurvival and proregenerative effects of cAMP to the transcriptional upregulation of Arg1 (Cai et al., 2002; Gao et al., 2004).

To identify chemical modulators of Arg1 expression that could not only be used to develop novel therapeutics, but also to develop new insight into regulation of the regenerative genetic program, we screened a well known library of clinically approved compounds, assembled originally by the NINDS, against a pool of cell clones expressing an Arg1 promoter-reporter construct. In completing our screen, we used an unusual methodological fea- 
ture that is important to highlight. While the initial screens to define true positives (those agents that induced the reporter and Arg1 protein levels) were performed in the Ratan Laboratory at Burke, the ability of these agents to overcome glial inhibitors was performed from a set of drugs containing true positives and false positives by an investigator in the Filbin laboratory at Hunter College. In carrying out these validation studies, the Hunter team was blinded to the properties of the compounds. Once the biological analysis was complete and true positives (e.g., daidzein) were identified, the findings were replicated (again, with the investigator blinded to the identity of the compound) in three other laboratories in three other institutions (Giger Laboratory at the University of Michigan, Ratan Laboratory at Burke, and Twiss Laboratory at the A.I. duPont Children's Hospital). Of note, despite using distinct sets of cell types and assays for these replication experiments, the concentration-response curves were virtually identical. Based on our experience, we would propose that translational efforts to identify novel drugs include a similar replication strategy in advance of publication (Steward et al., 2008).

Two critical pieces of evidence suggest that daidzein acts via the transcriptional upregulation of Arg1 to mediate the biological effects described herein. First, the canonical Arg1 inhibitor nor-NOHA completely abrogated the ability of daidzein to overcome inhibition of neurite growth by MAG, whereas nor-NOHA alone had no effect (Fig. 2G). Second, the ability of daidzein to render growth cones resistant to collapse by MAG was dependent on transcription (Fig. 3). Future studies using conditional knock-outs of Arg1 will examine the necessity of Arg1 for daidzein's effects in vivo.

Given that at least some of the effects of daidzein are mediated via the transcriptional induction of Arg1, an important unanswered question is how daidzein executes these functions. Our data suggests that Arg1 is induced by cAMPand CREB-independent mechanisms as intracellular cAMP levels and CREB phosphorylation were unchanged by daidzein treatment (Fig. 6A,B), indicating that daidzein is not activating adenylate cyclase, inhibiting PDEs, or engaging other CREBdependent pathways to induce Arg1 transcription. Daidzein has been evaluated in humans as an estrogen supplement and although these trials have been disappointing, they highlight an important biological property of isoflavones as agonists of the estrogen receptors, which are known transcriptional activators (Cassidy et al., 2006; Heldring et al., 2007). Daidzein has been reported to be neuroprotective to CNS neurons in glutamate excitotoxicity and oxygen/glucose deprivation models and to promote axonal outgrowth of hippocampal neurons on permissive substrates; these effects were attributed to the activity of daid-
$\mathrm{B}$

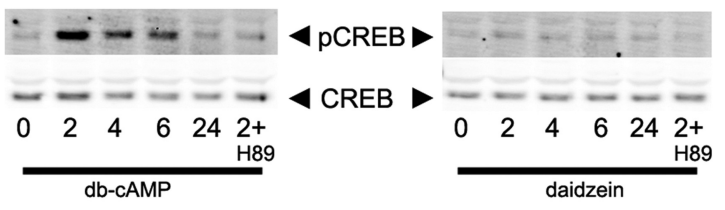

E
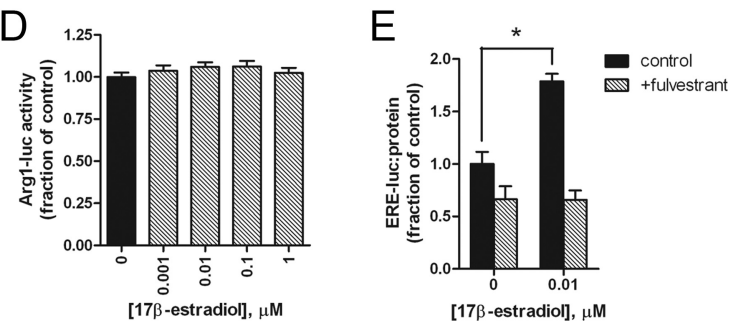

Figure 6. Daidzein does not increase CAMP levels or induce CREB phosphorylation but requires estrogen receptor binding to activate the Arg1 promoter. $\boldsymbol{A}$, Cortical neurons were treated with daidzein or db-CAMP (5 mM) for $24 \mathrm{~h}$ and then assayed for

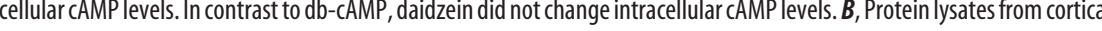
contrast, daidzein did not change pCREB levels at any time point. C, HT22 Arg1 reporter cells were treated with daidzein (20 $\mu \mathrm{m})$ $\boldsymbol{D}, \boldsymbol{E}, 17 \beta$-Estradiol alone $(\boldsymbol{D})$ is insufficient to activate the Arg1 promoter at concentrations that activate the ERE in HT22 cells $(\boldsymbol{E})$.

A

Figure 7. Daidzein protects neurons in two models of oxidative injury. Protein lysates from spinal cord motor neurons treated wh daidzein $(0-20 \mu \mathrm{m})$ for $24 \mathrm{~h}$ were immunoblotted to determine Arg1 protein levels. $A$, Representative Western blot of Arg1 (closed bars) or absence gonal bars) of BDNF. Omission of BDNF causes peroxynitrite-dependent motor neuron apoptosis, which was blocked by (20 $\mu \mathrm{m})$. C, Cortical neurons were treated with (diagonal bars) or without (closed bar) HCA, a compound that depletes intraneuronal glutathione and increases oxidative stress. Neurons treated with HCA undergo apoptosis, which is also blocked by daidzein $(20 \mu \mathrm{M})$. Data represent mean \pm SEM of $4-6$ independent experiments, ${ }^{*} p<0.05$ one-way ANOVA with NewmanKeuls post hoc test. zein on estrogen receptors (Zhao et al., 2002; Wang et al., 2008; Schreihofer and Redmond, 2009). While the induction of Arg1 by daidzein was estrogen receptor dependent, $17 \beta$-estradiol alone did not activate the Arg 1 promoter (Fig. $6 C, D$ ), indicating that although estrogenic activity is required, daidzein possesses additional activities that contribute to Arg1 induction. Accordingly, genistein, which is more estrogenic than daidzein, also failed to induce Arg1 protein levels. Future studies will exploit the chemical similarity between daidzein and genistein to identify the as yet unestablished activity of daidzein that endows regenerative capacities to an estrogen agonist. However, we cannot discern at this time whether the inability of genistein (which differs in one hydroxyl group from daidzein) to activate Arg1 relates to a positive activity of daidzein or a negative activity of genistein that is not present in daidzein.

In contrast to db-cAMP, neurons required priming with daidzein to overcome MAG inhibition in vitro. This difference reflects 
the biphasic response of neurons to cAMP in overcoming MAG inhibition, which consists of an immediate PKA-dependent and transcription-independent mechanism followed by a delayed PKA-independent activation of transcription (Qiu et al., 2002). As daidzein does not activate PKA, there is, as expected, no observed transcription independent phase to overcome glial inhibition. Thus priming with daidzein allows neurons to transcribe and accumulate effector proteins, such as Arg1, to resist glial inhibitors. Interestingly, daidzein did not increase axonal growth on permissive substrates (Fig. $2 \mathrm{H}, \mathrm{I}$ ), suggesting that the primary effect of daidzein may be to overcome external inhibitory cues (such as MAG). Additional factors that increase baseline growth, such as neurotrophins, may augment the effects of daidzein on neurons grown in an inhibitory environment; these combinational studies are ongoing.

Daidzein was effective in promoting axonal regeneration in an optic nerve crush model of CNS injury without lens damage in vivo. Though some optic nerve axons grew significant distances beyond the injury site, relatively few axons regenerated (Fig. 5), which is reminiscent of enhanced optic nerve regeneration from Rho inactivation, ROCK inhibition, and intraocular spermidine (Lehmann et al., 1999; Bertrand et al., 2005; Lingor et al., 2007; Deng et al., 2009). As such, we used the average length of the three longest axons as a measure of regeneration to highlight this property of daidzein. Of note, our lowest dose of subcutaneous daidzein was most effective, suggesting an U-shaped dose-response relationship and that our in vivo dosing protocols may require further optimization. Future studies will assess the proper dose/ duration of daidzein treatment to ensure that RGCs are exposed to the optimal amount of daidzein to see whether we can achieve the robust regeneration observed after manipulations such as inducing intraocular inflammation, oncomodulin injection, or genetic PTEN deletion (Yin et al., 2003, 2006; Park et al., 2008).

In this CNS injury model, puncture of the lens alone promotes significant regeneration, which has been attributed to a change in growth state of RGNs due to factors released by invading macrophages (Leon et al., 2000; Fischer et al., 2001; Yin et al., 2003, 2006). Lens injury also increases the efficacy of certain therapeutic manipulations (Fischer et al., 2004a,b). Whether lens puncture is required to enhance the number regenerating axons with daidzein is yet to be determined, though this possibility would limit its value for monotherapy. Interestingly, lens injury induces many gene expression changes, including the upregulation of Arg1, suggesting that daidzein may activate a similar transcriptional program (Fischer et al., 2004b). The mechanistic and biological congruence between lens injury and daidzein treatment may set the table for cotreatment with other therapeutic agents such as ROCK inhibitors.

Excitingly, daidzein was effective in vivo when given subcutaneously, indicating that it crossed the blood-brain barrier to enter the site of injury in the CNS. In agreement with our observation, one study detected daidzein in the brain of rats receiving an enteral mixture of soy proteins (Gu et al., 2005). However, we have not ruled out the possibility of a peripheral action of daidzein, such as a global increase in polyamine levels. Daidzein administered continuously for 2 weeks was more effective in promoting axon regeneration than an intraocular bolus injection following injury, suggesting a benefit for chronic application. The finding also suggests that regenerating axons may remain responsive to daidzein for extended periods postinjury. Future studies will determine the precise therapeutic window.

While daidzein is far more potent than analogs of cAMP in inducing Arg1 expression, the potency of daidzein for promoting survival and regeneration presented in this manuscript (Figs. $1 C$, $2 B, G-I, 3 E$ ) is unlikely to be sufficient for the compound to be effective as a drug in humans. With oral administration, daidzein and its metabolites (e.g., equol) rarely reach concentrations $>1$ $\mu \mathrm{M}$ (Nielsen and Williamson, 2007). However, even in the absence of a clear target, natural and synthetic analogs of daidzein are being developed that may enhance potency and efficacy to allow oral administration.

Despite these caveats, preliminary observations of subcutaneous daidzein initiated early following stroke in an animal model indicate that the unmodified daidzein can enhance functional recovery when behavior is analyzed 1 month after the ictus (Cho et al., unpublished observations). These promising observations suggest that human trials of daidzein given as soon as possible after the stroke or spinal cord injury and continued for weeks to months afterward may not be far off. The success of daidzein in more complex animal models of human disease could also provide affirmation of our Arglpromoter-reporter screen for identifying a new generation of neuroprotective agents with extant regenerative properties.

\section{References}

Baptiste DC, Fehlings MG (2007) Update on the treatment of spinal cord injury. Prog Brain Res 161:217-233.

Bertrand J, Winton MJ, Rodriguez-Hernandez N, Campenot RB, McKerracher L (2005) Application of Rho antagonist to neuronal cell bodies promotes neurite growth in compartmented cultures and regeneration of retinal ganglion cell axons in the optic nerve of adult rats. J Neurosci 25:1113-1121.

Block F, Tondar A, Schmidt W, Schwarz M (1997) Delayed treatment with rolipram protects against neuronal damage following global ischemia in rats. Neuroreport 8:3829-3832.

Cafferty WB, Strittmatter SM (2006) The Nogo-Nogo receptor pathway limits a spectrum of adult CNS axonal growth. J Neurosci 26: 12242-12250.

Cai D, Shen Y, De Bellard M, Tang S, Filbin MT (1999) Prior exposure to neurotrophins blocks inhibition of axonal regeneration by MAG and myelin via a cAMP-dependent mechanism. Neuron 22:89-101.

Cai D, Deng K, Mellado W, Lee J, Ratan RR, Filbin MT (2002) Arginase I and polyamines act downstream from cyclic AMP in overcoming inhibition of axonal growth MAG and myelin in vitro. Neuron 35:711-719.

Cassidy A, Albertazzi P, Lise Nielsen I, Hall W, Williamson G, Tetens I, Atkins S, Cross H, Manios Y, Wolk A, Steiner C, Branca F (2006) Critical review of health effects of soyabean phyto-oestrogens in post-menopausal women. Proc Nutr Soc 65:76-92.

Choi SY, Ha TY, Ahn JY, Kim SR, Kang KS, Hwang IK, Kim S (2008) Estrogenic activities of isoflavones and flavones and their structure-activity relationships. Planta Med 74:25-32.

Davis TG, Peterson JJ, Kou JP, Capper-Spudich EA, Ball D, Nials AT, Wiseman J, Solanke YE, Lucas FS, Williamson RA, Ferrari L, Wren P, Knowles RG, Barnette MS, Podolin PL (2009) The identification of a novel phosphodiesterase 4 inhibitor, 1-ethyl-5-\{5-[(4-methyl-1-piperazinyl)methyl]1,3,4-oxadiazol-2-yl $\}-N$-(tetrahydro-2 $H$-pyran-4-yl)- $H$-pyrazolo[3,4- $b]$ pyridin-4-amine (EPPA-1), with improved therapeutic index using pica feeding in rats as a measure of emetogenicity. J Pharmacol Exp Ther 330:922-931.

Dawson VL, Dawson TM, London ED, Bredt DS, Snyder SH (1991) Nitric oxide mediates glutamate neurotoxicity in primary cortical cultures. Proc Natl Acad Sci U S A 88:6368-6371.

DeMarch Z, Giampà C, Patassini S, Martorana A, Bernardi G, Fusco FR (2007) Beneficial effects of rolipram in a quinolinic acid model of striatal excitotoxicity. Neurobiol Dis 25:266-273.

DeMarch Z, Giampà C, Patassini S, Bernardi G, Fusco FR (2008) Beneficial effects of rolipram in the R6/2 mouse model of Huntington's disease. Neurobiol Dis 30:375-387.

Deng K, He H, Qiu J, Lorber B, Bryson JB, Filbin MT (2009) Increased synthesis of spermidine as a result of upregulation of arginase I promotes axonal regeneration in culture and in vivo. J Neurosci 29:9545-9552.

Esch F, Lin KI, Hills A, Zaman K, Baraban JM, Chatterjee S, Rubin L, Ash DE, 
Ratan RR (1998) Purification of a multipotent antideath activity from bovine liver and its identification as arginase: nitric oxide-independent inhibition of neuronal apoptosis. J Neurosci 18:4083-4095.

Estévez AG, Sahawneh MA, Lange PS, Bae N, Egea M, Ratan RR (2006) Arginase 1 regulation of nitric oxide production is key to survival of trophic factor-deprived motor neurons. J Neurosci 26:8512-8516.

Filbin MT (2003) Myelin-associated inhibitors of axonal regeneration in the adult mammalian CNS. Nat Rev Neurosci 4:703-713.

Fischer D, Heiduschka P, Thanos S (2001) Lens-injury-stimulated axonal regeneration throughout the optic pathway of adult rats. Exp Neurol 172:257-272.

Fischer D, He Z, Benowitz LI (2004a) Counteracting the Nogo receptor enhances optic nerve regeneration if retinal ganglion cells are in an active growth state. J Neurosci 24:1646-1651.

Fischer D, Petkova V, Thanos S, Benowitz LI (2004b) Switching mature retinal ganglion cells to a robust growth state in vivo: gene expression and synergy with RhoA inactivation. J Neurosci 24:8726-8740.

Gao Y, Deng K, Hou J, Bryson JB, Barco A, Nikulina E, Spencer T, Mellado W, Kandel ER, Filbin MT (2004) Activated CREB is sufficient to overcome inhibitors in myelin and promote spinal axon regeneration in vivo. Neuron 44:609-621.

Gong B, Vitolo OV, Trinchese F, Liu S, Shelanski M, Arancio O (2004) Persistent improvement in synaptic and cognitive functions in an Alzheimer mouse model after rolipram treatment. J Clin Invest 114:1624-1634.

Gray MJ, Poljakovic M, Kepka-Lenhart D, Morris SM Jr (2005) Induction of arginase I transcription by IL-4 requires a composite DNA response element for STAT6 and C/EBPbeta. Gene 353:98-106.

Gu L, Laly M, Chang HC, Prior RL, Fang N, Ronis MJ, Badger TM (2005) Isoflavone conjugates are underestimated in tissues using enzymatic hydrolysis. J Agric Food Chem 53:6858-6863.

Hall JM, McDonnell DP (1999) The estrogen receptor beta-isoform (ERbeta) of the human estrogen receptor modulates ERalpha transcriptional activity and is a key regulator of the cellular response to estrogens and antiestrogens. Endocrinology 140:5566-5578.

Hannila SS, Filbin MT (2008) The role of cyclic AMP signaling in promoting axonal regeneration after spinal cord injury. Exp Neurol 209:321-332.

Heemskerk J, Tobin AJ, Bain LJ (2002) Teaching old drugs new tricks. Meeting of the Neurodegeneration Drug Screening Consortium, 7-8 April 2002, Washington, DC. Trends Neurosci 25:494-496.

Heldring N, Pike A, Andersson S, Matthews J, Cheng G, Hartman J, Tujague M, Ström A, Treuter E, Warner M, Gustafsson JA (2007) Estrogen receptors: how do they signal and what are their targets. Physiol Rev 87:905-931.

Kato H, Araki T, Itoyama Y, Kogure K (1995) Rolipram, a cyclic AMPselective phosphodiesterase inhibitor, reduces neuronal damage following cerebral ischemia in the gerbil. Eur J Pharmacol 272:107-110.

Lange PS, Langley B, Lu P, Ratan RR (2004) Novel roles for arginase in cell survival, regeneration, and translation in the central nervous system. J Nutr 134:2812S-2817S; discussion 2812S-2817S.

Lehmann M, Fournier A, Selles-Navarro I, Dergham P, Sebok A, Leclerc N, Tigyi G, McKerracher L (1999) Inactivation of Rho signaling pathway promotes CNS axon regeneration. J Neurosci 19:7537-7547.

Leon S, Yin Y, Nguyen J, Irwin N, Benowitz LI (2000) Lens injury stimulates axon regeneration in the mature rat optic nerve. J Neurosci 20:46154626.

Lingor P, Teusch N, Schwarz K, Mueller R, Mack H, Bähr M, Mueller BK (2007) Inhibition of Rho kinase (ROCK) increases neurite outgrowth on chondroitin sulphate proteoglycan in vitro and axonal regeneration in the adult optic nerve in vivo. J Neurochem 103:181-189.

Lu P, Yang H, Jones LL, Filbin MT, Tuszynski MH (2004) Combinatorial therapy with neurotrophins and cAMP promotes axonal regeneration beyond sites of spinal cord injury. J Neurosci 24:6402-6409.

Mehta NR, Lopez PH, Vyas AA, Schnaar RL (2007) Gangliosides and Nogo receptors independently mediate myelin-associated glycoprotein inhibition of neurite outgrowth in different nerve cells. J Biol Chem 282:2787527886.

Mukhopadhyay G, Doherty P, Walsh FS, Crocker PR, Filbin MT (1994) A novel role for myelin-associated glycoprotein as an inhibitor of axonal regeneration. Neuron 13:757-767.

Neumann S, Bradke F, Tessier-Lavigne M, Basbaum AI (2002) Regeneration of sensory axons within the injured spinal cord induced by intraganglionic cAMP elevation. Neuron 34:885-893.

Nielsen IL, Williamson G (2007) Review of the factors affecting bioavailability of soy isoflavones in humans. Nutr Cancer 57:1-10.

Nikulina E, Tidwell JL, Dai HN, Bregman BS, Filbin MT (2004) The phosphodiesterase inhibitor rolipram delivered after a spinal cord lesion promotes axonal regeneration and functional recovery. Proc Natl Acad Sci U S A 101:8786-8790.

Park KK, Liu K, Hu Y, Smith PD, Wang C, Cai B, Xu B, Connolly L, Kramvis I, Sahin M, He Z (2008) Promoting axon regeneration in the adult CNS by modulation of the PTEN/mTOR pathway. Science 322:963-966.

Pearse DD, Pereira FC, Marcillo AE, Bates ML, Berrocal YA, Filbin MT, Bunge MB (2004) cAMP and Schwann cells promote axonal growth and functional recovery after spinal cord injury. Nat Med 10:610-616.

Qiu J, Cai D, Dai H, McAtee M, Hoffman PN, Bregman BS, Filbin MT (2002) Spinal axon regeneration induced by elevation of cyclic AMP. Neuron 34:895-903.

Ratan RR, Noble M (2009) Novel multi-modal strategies to promote brain and spinal cord injury recovery. Stroke 40:S130-132.

Ratan RR, Ryu H, Lee J, Mwidau A, Neve RL (2002) In vitro model of oxidative stress in cortical neurons. Methods Enzymol 352:183-190.

Rossignol S, Schwab M, Schwartz M, Fehlings MG (2007) Spinal cord injury: time to move? J Neurosci 27:11782-11792.

Sasaki T, Kitagawa K, Omura-Matsuoka E, Todo K, Terasaki Y, Sugiura S, Hatazawa J, Yagita Y, Hori M (2007) The phosphodiesterase inhibitor rolipram promotes survival of newborn hippocampal neurons after ischemia. Stroke 38:1597-1605.

Savitz SI, Fisher M (2007) Future of neuroprotection for acute stroke: in the aftermath of the SAINT trials. Ann Neurol 61:396-402.

Schreihofer DA, Redmond L (2009) Soy phytoestrogens are neuroprotective against stroke-like injury in vitro. Neuroscience 158:602-609.

Spina D (2008) PDE4 inhibitors: current status. Br J Pharmacol 155: 308-315.

Steward O, Sharp K, Yee KM, Hofstadter M (2008) A re-assessment of the effects of a Nogo-66 receptor antagonist on regenerative growth of axons and locomotor recovery after spinal cord injury in mice. Exp Neurol 209:446-468.

Twiss JL, Smith DS, Chang B, Shooter EM (2000) Translational control of ribosomal protein L4 mRNA is required for rapid neurite regeneration. Neurobiol Dis 7:416-428.

Venkatesh K, Chivatakarn O, Sheu SS, Giger RJ (2007) Molecular dissection of the myelin-associated glycoprotein receptor complex reveals cell typespecific mechanisms for neurite outgrowth inhibition. J Cell Biol 177: 393-399.

Wang P, Jeng CJ, Chien CL, Wang SM (2008) Signaling mechanisms of daidzein-induced axonal outgrowth in hippocampal neurons. Biochem Biophys Res Commun 366:393-400.

Willis DE, van Niekerk EA, Sasaki Y, Mesngon M, Merianda TT, Williams GG, Kendall M, Smith DS, Bassell GJ, Twiss JL (2007) Extracellular stimuli specifically regulate localized levels of individual neuronal mRNAs. J Cell Biol 178:965-980.

Yang L, Calingasan NY, Lorenzo BJ, Beal MF (2008) Attenuation of MPTP neurotoxicity by rolipram, a specific inhibitor of phosphodiesterase IV. Exp Neurol 211:311-314.

Yin Y, Cui Q, Li Y, Irwin N, Fischer D, Harvey AR, Benowitz LI (2003) Macrophage-derived factors stimulate optic nerve regeneration. J Neurosci 23:2284-2293.

Yin Y, Henzl MT, Lorber B, Nakazawa T, Thomas TT, Jiang F, Langer R, Benowitz LI (2006) Oncomodulin is a macrophage-derived signal for axon regeneration in retinal ganglion cells. Nat Neurosci 9:843-852.

Zhao L, Chen Q, Diaz Brinton R (2002) Neuroprotective and neurotrophic efficacy of phytoestrogens in cultured hippocampal neurons. Exp Biol Med (Maywood) 227:509-519. 